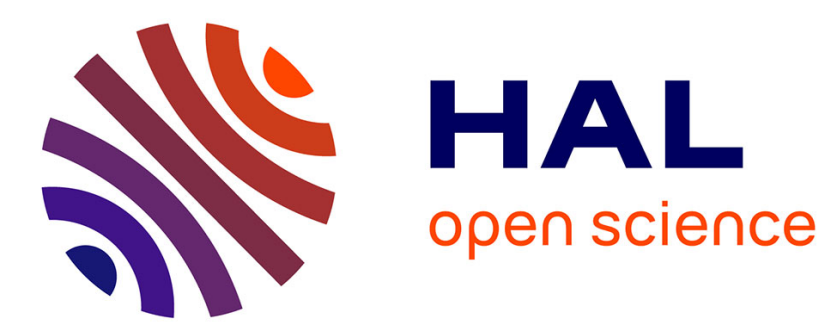

\title{
Dynamics of massive systems and synthesis of superheavy elements
}

Y. Abe, A. Marchix, Caiwan Shen, B. Yilmaz, G. Kossenko, D. Boilley, B.G. Giraud

\section{- To cite this version:}

Y. Abe, A. Marchix, Caiwan Shen, B. Yilmaz, G. Kossenko, et al.. Dynamics of massive systems and synthesis of superheavy elements. 13th Nuclear Physics Workshop "Marie and Pierre Curie" Pairing and Beyond - 50 years of the BCS Model, Sep 2006, Kazimierz Dolny, Poland. pp.491-501, 10.1142/S0218301307005922 . in2p3-00112874

\section{HAL Id: in2p3-00112874 https://hal.in2p3.fr/in2p3-00112874}

Submitted on 10 Nov 2006

HAL is a multi-disciplinary open access archive for the deposit and dissemination of scientific research documents, whether they are published or not. The documents may come from teaching and research institutions in France or abroad, or from public or private research centers.
L'archive ouverte pluridisciplinaire $\mathbf{H A L}$, est destinée au dépôt et à la diffusion de documents scientifiques de niveau recherche, publiés ou non, émanant des établissements d'enseignement et de recherche français ou étrangers, des laboratoires publics ou privés. 
International Journal of Modern Physics E

(C) World Scientific Publishing Company

\title{
Dynamics of Massive Systems and Synthesis of Superheavy Elements
}

\author{
YASUHISA ABE \\ Research Center for Nuclear Physics, Osaka University, \\ 10-1, Mihogaoka, Ibaraki City, Osaka 567-0047 Japan \\ abey@rcnp.osaka-u.ac.jp \\ ANTHONY MARCHIX \\ GANIL, BP 55027, 14076 Caen cedex 5, France \\ marchix@ganil.fr \\ CAIWAN SHEN \\ School of Science, Huzhou Teachers College, \\ Huzhou 313000, Zejiang, China \\ Center of Theoretical Nuclear Physics, National Laboratory of HIC, \\ Lanzhou 730000, China \\ cwshen@hutc.zj.cn \\ BULENT YILMAZ \\ Physics Department, Ankara University \\ Tandogan, Ankara, 06100, Turkey \\ bulent.yilmaz@science.ankara.edu.tr \\ GRIGORI KOSENKO \\ Department of Physics, Omsk University, \\ RU-644077 Omsk, Russia \\ kosenko@phys.omsu.omskreg.ru \\ DAVID BOILLEY \\ GANIL, BP 55027, 14076 Caen cedex 5, France \\ boilley@ganil.fr \\ BERTRAND G. GIRAUD \\ Service de Physique Theorique, CEA-Saclay, \\ Orme des Merisiers, Gif-sur-Yvette, 91191 France \\ bertrand.giraud@cea.fr \\ Received (received date) \\ Revised (revised date)
}

For the synthesis of superheavy elements, it is indispensable to divide the fusion process into two steps : Overcoming the Coulomb barrier and passing over the conditional saddle or the ridgeline. To facilitate the understanding of the mechanism which explains the 
fusion hindrance, we first employ an analytic model with an inverted parabola for the saddle. Then, results by realistic calculations are given for the cold fusion. Ambiguities of the model are also discussed for future investigations. Since the model is general, it is applied to incident channels with neutron-rich projectiles and/or targets. These are necessary for synthesis of nucleides in so-called superheavy island around $\mathrm{Z}=114$ and $\mathrm{N}=184$.

\section{Introduction}

A synthesis of superheavy elements becomes difficult as atomic numbers of the corresponding compound nuclei become larger. This is observed in the decreasing residue cross sections ${ }^{1}$. Therefore, reliable theoretical predictions are strongly desired on favourable incident channels and optimum incident energies. For that purpose, we firstly have to understand reaction mechanisms which result in such small residue cross sections ${ }^{2}$. There are two aspects : fragility of the atomic nuclei of the superheavy elements and hindrance of fusion in massive systems. The former is naturally understood, because the fission barrier of the Liquid Drop Model (LDM) is nearly equal to zero due to the fissility which is nearly equal to 1 , and thus, the compound nuclei are stabilized by just the shell correction energy or more generally by the quantum-mechanical microscopic energy. The latter aspect, the fusion hindrance, has been well observed experimentally, but is not yet well understood physically in its reaction mechanism. The present authors have proposed a two-step model for the fusion process in massive systems, i.e., for the fusion hindrance ${ }^{3,4}$. In section 2 , we briefly recall the two-step model. The mechanism of the fusion hindrance is clearly explained by the analytic model in section $3^{5}$. As examples of the realistic calculations, we discuss the ${ }^{56} \mathrm{Fe}+{ }^{208} \mathrm{~Pb}$ system, as well as systems including ${ }^{132} \mathrm{Sn}$ as target and/or projectile. Remarks are given on remaining ambiguities in realizations of the model which are subjects for future investigations. A tentative conclusion is also given.

\section{Two-Step Model for Fusion Hindrance}

The model is based on the essential observation that contact configurations of massive incident projectiles and targets are mostly located outside of the corresponding conditional saddle point. Indeed, the deformations of the dinuclear configurations are naturally very large, whereas the deformations of the conditional saddle points are small due to the large fissility parameter for the compound nuclei ${ }^{2}$. This means that after an incident system overcomes the Coulomb barrier to contact, the system has to overcome once more the conditional saddle or the ridgeline. As is well known in Deep-Inelastic Collisions (DIC $)^{6}$, the incident systems are supposed to loose their incident kinetic energy when they pass over the Coulomb barrier, and/or during the formation of the stuck di-nucleus systems. Thus, the systems do not have enough kinetic energy for overcoming the saddle point, and would fuse only by the fluctuation associated with the energy dissipation. Therefore, the probability for overcoming the saddle point, i.e., for the formation of the spherical compound 
nuclei is extremely small ${ }^{7}$. This is the main physical mechanism explaining the hindrance. Of course, the extent of the hindrance depends on the mass asymmetry of the incident channel, even though the compound nucleus formed is the same. This is readily elucidated by the LDM energy surface in two dimensional space with the distance parameter between projectile and target, and their mass asymmetry in the Two-Center parametrization, schematically shown in Fig. 1. In a large mass asymmetry system like ${ }^{48} \mathrm{Ca}+{ }^{244} \mathrm{Pu}$, the contact point is very close to or even inside the conditional saddle point or the ridgeline, while in a small asymmetry case like ${ }^{86} \mathrm{Kr}+{ }^{208} \mathrm{~Pb}$, the contact point is far outside the ridgeline. Thus, the incident channel with a large mass asymmetry has almost no need to overcome the ridgeline, though the system may return back to re-separation with a substantial probability before sliding down to the spherical compound. On the other hand, the system with a small asymmetry has to climb up over the saddle point, mainly by energy fluctuation. The difficulty in climbing this additional "barrier" is amplified by the dissipative dynamics, as it is explained analytically in the next section.

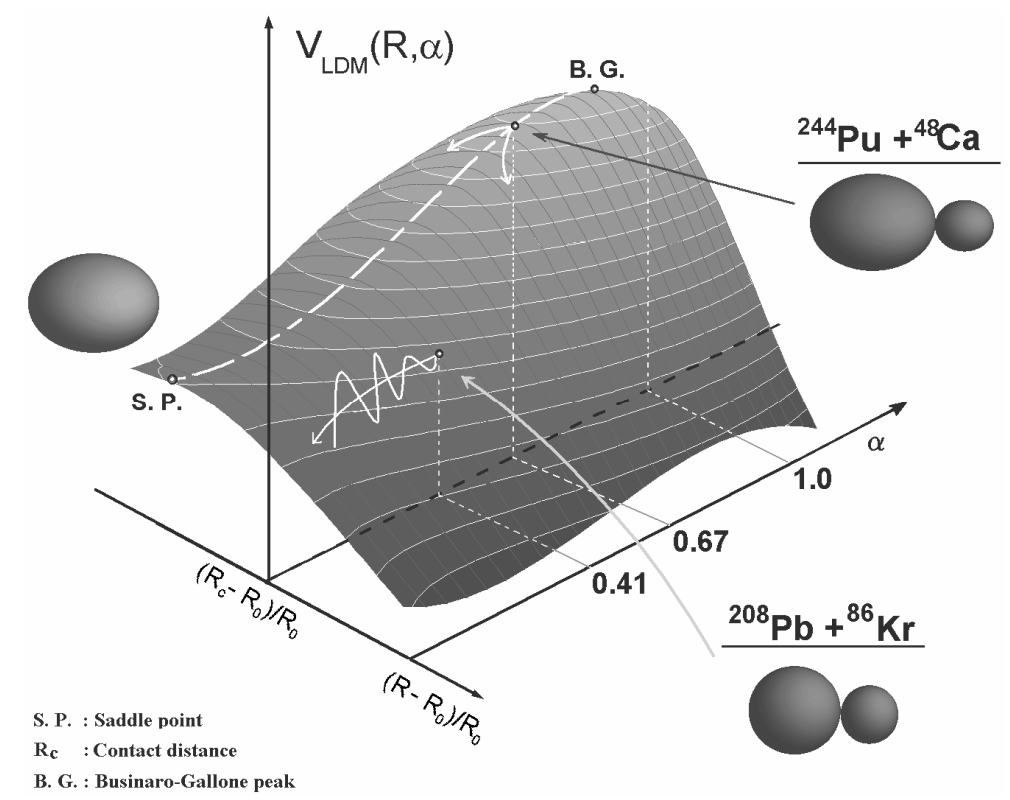

Fig. 1. A schematic illustration of relative position between contact point $R_{c}$ and ridgeline(shown with a long dash-dotted line) as a function of mass asymmetry. $R$ and $R_{0}$ in the x-axis denotes the distance between two centers of the potentials and the radius of the spherical compound nucleus used as a normalization. $\alpha$ of the y-axis is the mass asymmetry of the dinuclear configuration given by the incident channel. Typical trajectories are shown schematically for the cases with a large mass asymmetry case and a small mass asymmetry. In the latter, most of the trajectories are reflected to re-separation, i.e., to quasi-fission channels, while in the former, they bifurcate into two directions with a substantial probability for formation of the compound nucleus. 


\section{Analytic Model for Fusion Hindrance}

In order to understand the mechanism of the fusion hindrance, we employ a simple one-dimensional model for overcoming the saddle point under dissipation, i.e., we approximate the saddle point as an inverted parabola and assume the inertial mass and the friction coefficient to be constant around the saddle point ${ }^{5}$. Then, equations to be solved are as follows,

$$
\begin{aligned}
& \frac{d q}{d t}=\frac{1}{\mu} p, \\
& \frac{d p}{d t}=\mu \omega^{2} q-\beta p+R(t),
\end{aligned}
$$

where $\mu$ denotes the inertial mass for the motion of the distance $q$ between the components of the di-nucleus, and $\omega$ is the frequency of the inverted parabola. $\beta$ denotes the reduced friction $\gamma / \mu$, while $R(t)$ represents the fluctuation force associated with the friction $\gamma$, which is assumed to be Gaussian and to satisfy the dissipation-fluctuation theorem,

$$
<R(t) R\left(t^{\prime}\right)>=2 \gamma T \delta\left(t-t^{\prime}\right),
$$

where the memory effect is neglected ${ }^{8}$ and $T$ is the temperature of the heat bath, i.e., that of the compound nucleus. The symbol $<>$ denotes the average over all the possible realizations of $R(t)$. Eq.(1) is a linear equation, and thus an analytic solution can be written as a function of the parameters in the equation and initial values of the coordinate $q_{0}$ and the associated momentum $p_{0}$. The probability distribution function in the phase space $(q, p)$ at any time $t$ is naturally Gaussian around the mean trajectory. By integrating over the whole p-space and half q-space, we obtain the probability for the system to enter into the other side of the saddle point, and then, letting the time $t$ become infinite, we obtain the probability for the formation of the compound nucleus. It is given simply by an error function as follows,

$$
F\left(p_{0}, q_{0}\right)=\frac{1}{2} \operatorname{erfc}\left[\sqrt{\frac{\eta+\eta^{\prime}}{2 \eta}}\left[\sqrt{\frac{\mathrm{B}}{\mathrm{T}}}-\frac{1}{\eta+\eta^{\prime}} \sqrt{\frac{\mathrm{K}}{\mathrm{T}}}\right]\right],
$$

where $B=\mu \omega^{2} q_{0}^{2} / 2$ and $K=p_{0}^{2} / 2 \mu$. The former denotes the relative height of the saddle point measured from the contact configuration, while the latter is the initial kinetic energy at the contact point, i.e., the energy left after overcoming the Coulomb barrier where friction is supposed to be also in action to heat up the system. The non-dimensional parameter $\eta$ is defined as $\beta / 2 \omega$ and $\eta^{\prime}$ is $\sqrt{1+\eta^{2}}$. The hindrance is readily understood from Eq.(3). In order for the formation probability to be $1 / 2$, the argument of the error function should be equal to zero. Then, a necessary remaining kinetic energy is given as follows,

$$
K=\left(\eta+\eta^{\prime}\right)^{2} B
$$

This means that the remaining kinetic energy at the contact point should be much larger than the saddle point height, because the factor in front of $B$ in the r.h.s. 
is estimated to be ten or a few tens, from the strength of the friction calculated with one-body Wall-and-Window Formula (WWF). ${ }^{9}$ This is consistent with other experiments, such as measured fission time scale ones ${ }^{10,11}$. Thus, it is apparent that a large extra kinetic energy is required to overcome the hindrance of fusion. It is worth to notice once more here that the kinetic energy $K$ is not the incident kinetic energy, but the remaining one at the contact point ; it is reduced very much from the incident c.m. energy if we employ the Surface Friction Model (SFM) ${ }^{6}$ for the description of the first step, i.e., for the process of passing over the Coulomb barrier. At the same time, the kinetic energy at the contact point has a distribution due to the fluctuation associated to the surface friction, which is Gaussian due to the assumption of a Gaussian nature for the fluctuation force. Its mean value is obtained to be zero in massive systems leading to superheavy elements ${ }^{12}$. This means that for a given incident energy, the kinetic energy is completely dissipated on the average, and thus, the probability for the system to have enough energy to overcome the saddle point is very small. By taking an average of the formation probability $F\left(p_{0}, q_{0}\right)$ over the distribution of $p_{0}$, we obtain the formation probability for a given incident energy, again in an error function form as follows ${ }^{5}$,

$$
P_{\text {form }}=\frac{1}{2} \operatorname{erfc}\left[\sqrt{\frac{\mathrm{B}}{\mathrm{T}}}\right] .
$$

Since the mean kinetic energy is equal to zero, the formation is only possible in the tail of the momentum distribution, i.e., by the fluctuation. This is exactly equal to the expression used by Swiatecki et al. ${ }^{13}$ as a diffuse factor. For the calculations of $B$, they introduced a parameter $s$ for the separation between two nuclear surfaces where the evolution of the di-nucleus system starts. The value of $s=1.6 \mathrm{fm}$ that they take in order to reproduce the observed residue cross sections appears to be very large, probably unphysically too large, because the point is located even outside the Coulomb barrier. This is inferred to be due to the oversimplification of the dynamics to one-dimension, i.e., degrees of freedom other than the relative distance are completely frozen. Possible effects of other degrees of freedom can be investigated analytically within the multi-dimensional parabolic model ${ }^{5,14}$. Preliminary results with reasonable parameters show that the effective saddle point height is larger than that value $B$ in the one-dimensional model. In our realistic calculations below, the formation probability is calculated not by a one-dimensional model, but by a two-dimensional model with a coordinate-dependent inertial mass and a friction coefficient, and with the LDM potential surface like the one shown in Fig.1. The mass is calculated by the Werner-Wheeler method ${ }^{15}$, and the friction by WWF, while the potential results from the finite range $\mathrm{LDM}^{16}$ with the Two-Center parametrization ${ }^{17}$. There is no free parameter in the model, but the two-dimensional model with the distance and the mass asymmetry itself assumes other degrees of freedom to be frozen. The most important one would be the socalled neck degree of freedom, which will be taken into account in the near future, but is treated as a free parameter in the present investigations. 
It is worth to emphasize that the two-step model provides a method for a connection between a two-body collision process and the subsequent one-body shape evolution. This should be called as a "statistical connection" (This is completely different from those discussed before, say, the adiabatic, or the diabatic connection. $)^{4}$. For, the remaining kinetic energy at the contact point has a distribution, which is an initial condition for the dynamics of the second step. It is also worth to notice here that one cannot connect the two potentials (potential of two body collisions and potential energy surface of the two-center parametrization) at the contact point, because they have different physical definitions. As is well known in cluster model studies, the energy surface of the composite system is the total energy including the kinetic energy of collective motions in an approximate way, say, the kinetic energy of the radial motion, while the potential in the two-body collisions naturally does not include the kinetic energy.

\section{Practical Realizations; Comparisons with experiments}

In the two-step model, the so-called sticking probability has to be calculated, which is just the probability of passing over the Coulomb barrier. In lighter heavy-ions, this is taken as a transmission coefficient or a barrier penetration factor, but in massive systems, the system is supposed to be dissipative and thus, the probability is reduced. The mean trajectory starting with a given incident energy well above the Coulomb barrier may be even reflected by the Coulomb barrier due to the loss of the kinetic energy which is supposed to be transformed into the thermal energy of intrinsic nucleonic degrees of freedom. As is well known, the viewpoint of dissipative dynamics is successful for the description of DIC, say, the SFM. It would be better to readjust the parameters of the model to accommodate the lower energy region of fusion into the superheavy elements. And refinements such as inclusion of the rolling friction etc. would be necessary, but we do not change anything in the present preliminary investigation. Note that we use it until incident systems reach the contact point, i.e., just for passing over the Coulomb barrier, and there we start a dissipation-fluctuation dynamics of shape evolution of the amalgamated system. As stated in the previous section, the distribution of the radial momentum at the contact point is a Gaussian, i.e., a Boltzmann distribution with the temperature of the heat bath; and the sticking probability is obtained by the ratio between the number of trajectories that reach the contact point and the total number of the trajectories. Details are already published elsewhere ${ }^{4,7}$.

In our model, calculations are performed for each total spin $\mathrm{J}$ and then cross sections are obtained by summation over J, which is different from Swiatecki et al. ${ }^{13}$. The fusion probability is obtained for each $\mathrm{J}$ by a product of the two probabilities,

$$
P_{\text {fusion }}^{J}=P_{\text {stick }}^{J}\left(E_{\text {c.m. }}\right) P_{\text {form }}^{J}\left(E_{\text {c.m. }}\right),
$$

where $P_{\text {stick }}^{J}$ and $P_{\text {form }}^{J}$ are calculated by SFM and the two-dimensional Langevin equation with the distance and the mass asymmetry degrees of freedom, respec- 
tively, as stated above. With this probability, we calculate fusion excitation functions as usual,

$$
\sigma_{\text {fusion }}\left(E_{c . m .}\right)=\frac{\pi}{k^{2}} \sum_{J}(2 J+1) P_{\text {fusion }}^{J}\left(E_{c . m .}\right) .
$$

Furthermore, we calculate $x n$ residue excitation functions, combined with the survival probability $P_{x n}^{J}\left(E^{*}\right)$,

$$
\sigma_{x n}\left(E_{c . m .}\right)=\frac{\pi}{k^{2}} \sum_{J}(2 J+1) P_{f u s i o n}^{J}\left(E_{c . m .}\right) P_{x n}^{J}\left(E^{*}\right),
$$

where $E^{*}=E_{c . m .}+Q$, with the reaction Q-value calculated with P. Möller et al.'s table $^{18}$. This is calculated with a new statistical code, KEWPIE $\mathrm{II}^{19}$ suitable for the superheavy elements. The preliminary version is already published ${ }^{20}$, and a revised version which is used in the present calculations will be published soon. In the present calculations, we treat the neck parameter as a free parameter.

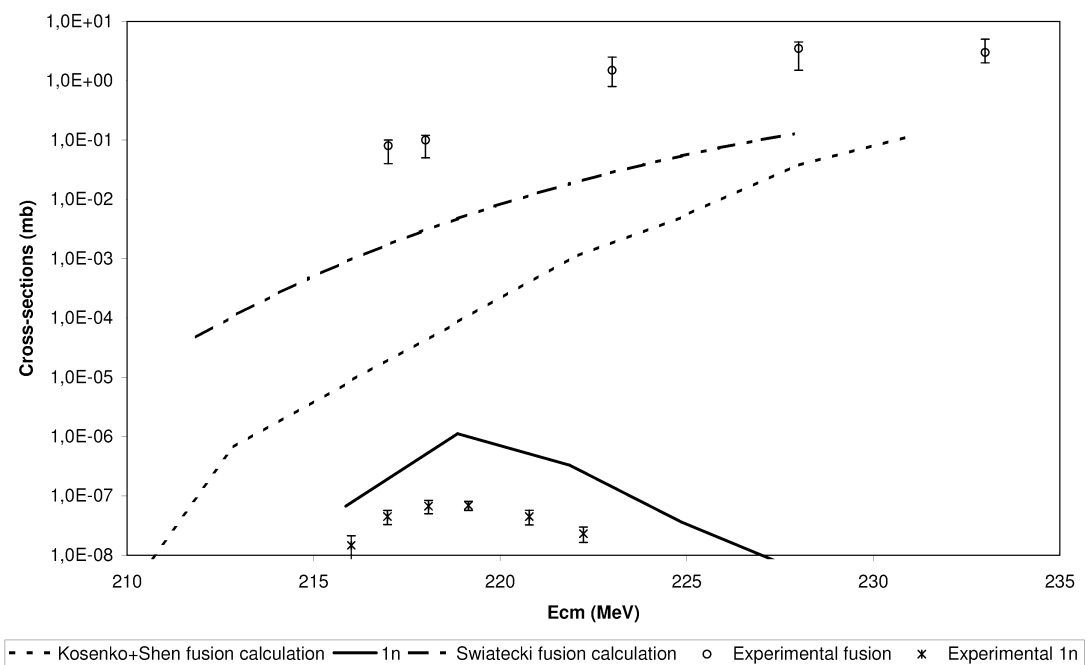

Fig. 2. Comparison of calculated fusion and $1 n$ residue cross sections with experimental data on ${ }^{58} \mathrm{Fe}+{ }^{208} \mathrm{~Pb}$.

Unfortunately, there are not so many experimental data on fusion excitation to be compared with our calculations. The ${ }^{58} \mathrm{Fe}+{ }^{208} \mathrm{~Pb}$ system is the only exception among the cold fusion systems ${ }^{21}$. Thus, in the present paper, we concentrate on this system. In Fig. 2, the fusion and $1 n$ excitation functions are compared with the experiments. Here we choose the neck parameter to be 0.6. The reproduction (full curve) of the $1 n$ excitation function by the Kosenko-Shen model is very good, considering the peak position as well as its absolute value. Note that the calculated values are not yet averaged over the width of the experimental incident c.m. energy. 
The fusion excitation functions are calculated with the two different models; one of them is the present two-step model, while the other is Swiatecki et al.'s model ${ }^{13}$. It is interesting to observe that the calculated fusion excitations (dash-dotted and dashed lines ) are far smaller than the experiment which, however, is apparently hindered. The fusion probability in the present calculations is the product of the sticking probability calculated with SFM and the formation probability calculated with a two-dimensional model. The other model gives the fusion probability by a product of so-called capture probability and diffuse factor. The latter factor is the same as the formation probability of a one-dimensional version of the multidimensional dissipation-fluctuation dynamics, as shown in Y. Abe et al. ${ }^{5}$, and is adjusted with a free parameter $s$ as discussed above.

The SFM sticking probability is much smaller than that of the empirical formula for the capture probability ${ }^{22}$ used by Swiatecki et al. ${ }^{13}$, but this is partially cancelled by the difference in the second factor, i.e., that between the formation probability and the diffuse factor. Of course, we cannot conclude at present which model is more realistic. This is because the quantitative accuracy of SFM is not sure, and as well we do not know how accurately the empirical formula of the capture cross section can be extrapolated to heavier systems, nor how the one-dimensional model with the parameter $s$ is justified.

In our previous calculations, we reproduced the fusion cross section with the neck parameter 0.8 , but could not reproduce the absolute value of the $1 n$ cross section, and had to introduce a reduction factor $1 / 2$ to the predicted shell correction energy $^{23}$. In brief, to reproduce the fusion and the $1 n$ cross section at the same time is difficult with the present model as well as in the model of Swiatecki et al.

Since the present theoretical framework is general, it can be applied to various incident systems, such as those including neutron-rich isotopes which are necessary to reach superheavy nucleides around the so-called superheavy island with $\mathrm{Z}=114$ and $\mathrm{N}=184$. As examples, we apply the present model to ${ }^{132} \mathrm{Sn}+{ }^{160} \mathrm{Gd}^{24}$ and ${ }^{132} \mathrm{Sn}+{ }^{132} \mathrm{Sn}$ systems. The latter system is mass symmetric, hence it is interesting to know how strong is the fusion hindrance. The results are shown in Fig. 3 , where we take the neck parameter to be zero arbitrarily. (If it is $\approx 0.8$, all the results are one to two orders of magnitude larger than those shown in the figure). Of course, there is no consideration of the possible special enhancement mechanisms due to a neutron $\operatorname{skin}^{25}$. Nevertheless, the present results show rather large fusion cross section, i.e., the hindrance is not very strong. And $x n$ residue cross sections are very large, because the survival probabilities are favoured by the neutron richness of the compound nuclei. That is, by using neutron rich targets and projectiles, we reach the compound nuclei which are located in the far-side of the $\beta$ stability line. This shows a crucial role played by neutron-rich projectiles and targets for the sysntheses of superheavy elements. Considering the ambiguity in the neck parameter which may give rise to much larger cross sections, we think that our results are encouraging for further study. 


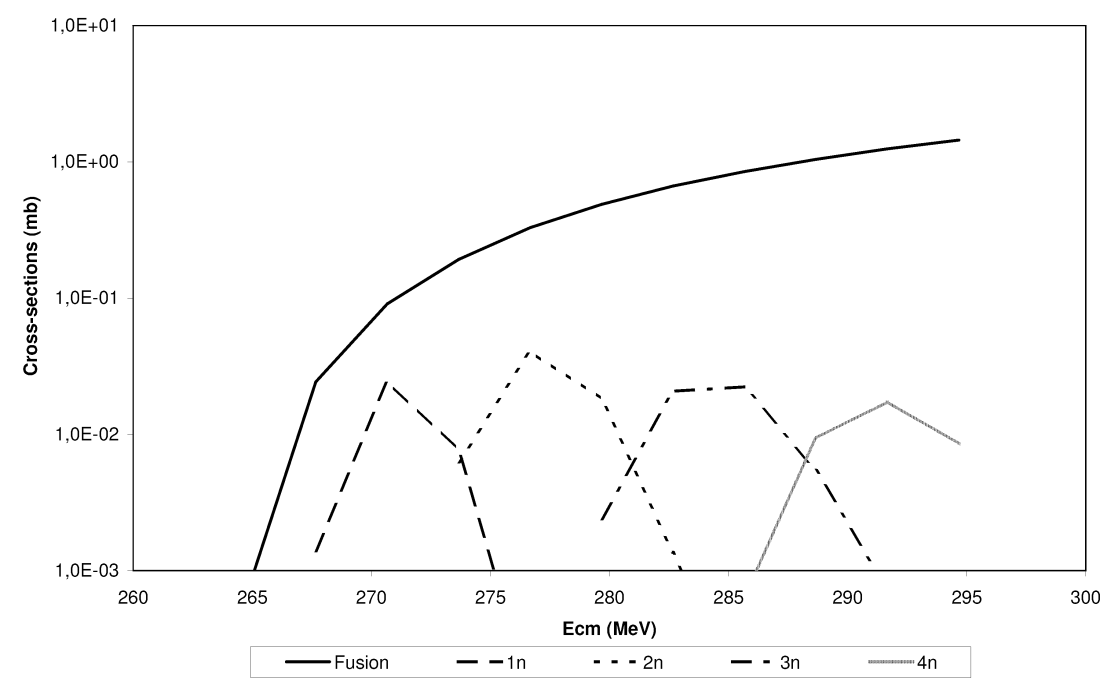

Fig. 3. Fusion and xn residue cross sections calculated with the two-step model and statistical code KEWPIE II.

\section{Discussion and tentative conclusion}

As stated above, there is a dilemma in reproducing the fusion and the residue excitation functions. Of course, there are aspects which are not yet properly taken into account. For example, the Kramers factor and the collective enhancement factor which act in an opposite way to each other in calculations of the fission probability, are crucial in calculations of the survival probability in the statistical decay. If such two factors finally result in an increase of fission width by two to three orders of magnitude, the dilemma might be solved. Another parameter to be mentioned is the shell damping energy, which is taken to be $18.5 \mathrm{MeV}$ as usual ${ }^{26}$. But we are not sure that the value could be accurate also in the superheavy nucleides where "shells" are much more fragile than those in lighter nuclei where the value is obtained.

Although there are still ambiguities in quantitative predictions as explained above, it can be stated that the two-step model with the statistical connection is canonical for the description of the fusion of massive systems and indispensable for a prediction of the synthesis of the superheavy elements. Efforts to eliminate such ambiguities are yet to be made for precise quantitative theoretical predictions on the synthesis of superheavy elements, which are under way both analytically with the simplified model and numerically with the realistic model.

\section{Acknowledgements}

One of the authors (Y.A.) acknowledges that the present work is supported by JSPS Grant No. 18540268. He also thanks GANIL, Huzhou University and Service 
de Physique Theorique, CEA-Saclay for providing him with the opportunities for working together with A. M. and D. B., C. S., and B. G. G., respectively. A. M. and D. B. acknowledge supports by JSPS which enabled them to work together with Y. A. at RCNP, Osaka Univ. last summer. B.Y. thanks the supports by TUBITAK which enabled him to work with Y. A. at RCNP, Osaka Univ. for six months. C. S. also acknowledges RCNP for the invitation supported by JSPS Grant No. 18540268. Part of C. S.' work is also supported by the National Natural Science Foundation of China under grant number 10675046 and Natural Science Foundation of Zhejiang Province, China under grant number Y605476. All the visitors of RCNP appreciate the hospitality of the members of the theory group of RCNP, extended to them during their stay.

\section{References}

1. S. Hofmann, Rep. Prog. Phys. 61, 639 (1998).

S. Hofmann and G. Muenzenberg, Rev. Mod. Phys. 72, 733 (2000).

K. Morita, et al., J. Phys. Soc. Japan 73, 1738 (2004).

2. Y. Abe, Eur. Phys. J. A13, 143 (2002).

3. Y. Abe, Proc. Symp. on Fusion Dynamics at the Extremes, Dubna 25-27, May, 2000 (World Scientific, Singapore, 2001) pp. 162-173.

4. C. Shen, G. Kosenko, and Y. Abe, Phys. Rev. C66, 061602 (2002). Y. Abe, C. Shen, G. Kosenko, and D. Boilley, Physics of Atomic Nuclei, 66, 1057 (2003).

5. Y. Abe, D. Boilley, B. G. Giraud, and T. Wada, Phys. Rev. E61, 1125 (2000).

D. Boilley, Y. Abe, and J.-D. Bao, Eur. Phys. J. A18, 627 (2003).

6. D. H. E. Gross and H. Kalinowski, Phys. Rep. 45, 175 (1978).

see also, C. F. Tsang, Physica Scripta Vol. 10A, 90 (1974), and D. Bangert and H. Freiesleben, Nucl. Phys. A340, 205 (1980).

7. Y. Abe, D. Boilley, G. Kosenko, et al., Prog. Theor. Phys. Suppl. 146, 104 (2002). Y. Abe, D. Boilley, G. Kosenko, and C. Shen, Acta. Phys. Pol. B34, 2091 (2003).

8. D. Boilley and Y. Lallouet, J. Stat. Phys. (2006).

9. J. Blocki, et al., Ann. Phys. (N.Y.) 113, 330 (1978).

10. T. Wada, Y. Abe, and N. Carjan, Phys. Rev. Lett. 70, 3538 (1993).

11. Y. Abe, et al., Phys. Rep. Nos. 2 and 3, (1996).

12. G. Kosenko, C. Shen, and Y. Abe, J. Nucl. Radiochem. Sci. 3, 19 (2002).

13. W. J. Swiatecki, et al., Acta Phys. Pol. B34, 2049 (2003) and Phys. Rev. C71, 014602 (2005).

14. B. Yilmaz and Y. Abe, private communication.

15. K. J. R. Davies and J. R. Nix, Phys. Rev. C14, 1977 (1976).

16. H. J. Krappe, J. R. Nix, and A. J. Sierk, Phys. Rev. C20, 992 (1979).

17. A. Iwamoto, et al., Prog. Theor. Phys. 55, 115 (1976).

K. Sato, et al., Z. Phys. A290, 145 (1979).

18. P. Moeller, et al., Atomic Data and Nuclear Data Tables 59, 185 (1995).

19. A. Marchix, D. Boilley and Y. Abe, a new version KEWPIE II under preparation for publication.

20. B. Bouriquet, Y. Abe, and D. Boilley, Comp. Phys. Comm. 159, 1 (2004).

21. M. G. Itkis, et al., Proc. Fusion Dynamics at the Extremes, 25-27, May 2000 ( World Scientific, Singapore, 2001) p.93. 
22. K. Siwek-Wilczynska, E. Siemaszko, and J. Wilczynski, Acta Phys. Pol. B33, 451 (2002) and Phys. Rev. C69, 0246141 (2004).

23. Y. Abe, B. Bouriquet, C. Shen, and G. Kosenko, Nucl. Phys. A722, 241c (2003)[ see also Erratum; Nucl. Phys. A733, 351 (2004)]

Y. Abe, B. Bouriquet, G. Kosenko, and C. Shen, Nucl. Phys. A734, 168 (2004)

B. Bouriquet, G. Kosenko, and Y. Abe, Prog. Theor. Phys. 154, 425 (2004), and Eur. Phys. J. bf A22, 9 (2004).

24. Y. Abe, et al., Physics of Atomic Nuclei, 69, 1101 (2006).

25. J. F. Liang, et al., Phys. Rev. Lett. 91, 152701 (2003).

26. A. V. Ignatyuk, et al., Sov. J. Nucl. Phys. 21, 255(1975). 